\title{
Kadec-Klee Property in Orlicz Function Spaces Equipped with S-Norms
}

\author{
Jiaqi Dong, ${ }^{1}$ Yunan Cui ${ }^{D},{ }^{1}$ and Marek Wisła ${ }^{2}$ \\ ${ }^{1}$ Department of Mathematics, Harbin University of Science and Technology, Harbin 150080, China \\ ${ }^{2}$ Faculty of Mathematics and Computer Science, Adam Mickiewicz University, Ul. Uniwersytetu Poznańskiego 4, \\ 61-614 Poznań, Poland \\ Correspondence should be addressed to Yunan Cui; cuiya@hrbust.edu.cn
}

Received 2 July 2021; Accepted 16 December 2021; Published 30 January 2022

Academic Editor: Seppo Hassi

Copyright (C) 2022 Jiaqi Dong et al. This is an open access article distributed under the Creative Commons Attribution License, which permits unrestricted use, distribution, and reproduction in any medium, provided the original work is properly cited.

Using some new techniques, the necessary and sufficient conditions for Kadec-Klee property of Orlicz function spaces equipped with s-norms are presented. An original method that was used in the process of inquiry and the obtained results also systematically complete and broaden the characterization of Kadec-Klee property of Orlicz spaces.

\section{Introduction and Preliminaries}

Orlicz spaces, introduced by W. Orlicz in 1932, form a wide class of Banach spaces of measurable functions (in the case of atomless measure) or sequences (in the case of counting measure) (see [1]). On Orlicz spaces of measurable functions, the classical Orlicz and Luxemburg norm can be defined by use of the Amemiya formula: $\|x\|_{\Phi}^{o}=\inf _{k>0}(1 / k)$ $\left(1+I_{\Phi}(k x)\right)$ and $\|x\|_{\Phi}=\inf _{k>0}(1 / k) \max \left\{1, I_{\Phi}(k x)\right\}$, respectively, where $\Phi$ is an Orlicz function and $I_{\Phi}$ $(x)=\int_{G} \Phi(x(t)) \mathrm{d} t$. Based on these statements, H. Hudzik and L. Maligrada introduced Orlicz spaces equipped with the p-Amemiya norms, where $p \in[1, \infty]$ and defined $\|x\|_{\Phi}^{p}=$ $\inf _{k>0}(1 / k)\left(1+I_{\Phi}^{p}(k x)\right)^{(1 / p)}$ in 2000 (see $\left.[2,3]\right)$. M. Wisła presented a universal and general method of introducing norms (s-norms) in Orlicz spaces in 2019, and the introduction of Orlicz spaces equipped with s-norms covers all the cases mentioned above.

1.1. Introduction. In the following, by $N$ we will denote the sets of natural numbers and $R$ and $R^{+}$the sets of real and nonnegative real numbers, respectively. By $S(X)$ and $B(X)$, we will denote the unit sphere and the unit ball of the Banach space $X$, respectively.
Let $(G, \Sigma, \mu)$ be a finite nonatomic measure space, and $L^{0}=L^{0}(\mu)$ be the set of all ( $\mu$-equivalence) classes of $\Sigma$-measurable real functions defined on $G$.

Definition 1. A continuous function $\Phi: R \longrightarrow R^{+}$is called convex if

$$
\Phi\left(\frac{u+v}{2}\right) \leq \frac{\Phi(u)+\Phi(v)}{2}
$$

for all $u, v \in R$. If, in addition, the two sides of formula (1) are not equal for all $u \neq v$, then we say $\Phi$ is strictly convex.

Definition 2. A function $\Phi: R \longrightarrow R^{+}$goes by name of an Orlicz function if $\Phi$ is nonnegative, even, convex satisfying $\Phi(0)=0$ and $\lim _{u \longrightarrow \infty}(\Phi(u) / u)=\infty$.

$\Psi(v)=\sup \{u|v|-\Phi(u): u \geq 0\}$ for all $v \in R$ is called the complementary function of $\Phi(u)$ in the sense of Young. Obviously, $\Psi$ is also an Orlicz function.

Definition 3. $I_{\Phi}$ is introduced as a modular of $\Phi$ by

$$
I_{\Phi}(u)=\int_{G} \Phi(u(t)) \mathrm{d} t
$$

for all $u \in L^{0}(\mu)$. 
Then, the Orlicz space $L_{\Phi}$ and its subspace $E_{\Phi}$ are generated by an Orlicz function and $\Phi$ are linear spaces of measurable functions defined by the following formulas:

$$
\begin{aligned}
& L_{\Phi}=\left\{u: I_{\Phi}(\lambda u)<\infty \text { for some } \lambda>0\right\} ; \\
& E_{\Phi}=\left\{u: I_{\Phi}(\lambda u)<\infty \text { for any } \lambda>0\right\} .
\end{aligned}
$$

For each $u \in L_{\Phi}$, let Luxemburg norm be defined by

$$
\|x\|_{\Phi}=\inf \left\{k>0, I_{\Phi}\left(\frac{x}{k}\right) \leq 1\right\},
$$

and Orlicz norm by

$$
\|x\|_{\Phi}^{o}=\inf _{k>0} \frac{1}{k}\left(1+I_{\Phi}(k x)\right) .
$$

Definition 4 (see [4]). A function $s:[0, \infty) \longrightarrow[1, \infty)$ will be called an outer function, if it is convex and

$$
\operatorname{Max}\{u, 1\} \leq s(u) \leq u+1 \text { forall } u \geq 0 .
$$

Definition 5 (see [4]). Let $s$ be an outer function and $\Phi$ be an Orlicz function. Then, the function

$$
\|x\|_{\Phi, s}=\inf _{k>0} \frac{1}{k} s\left(I_{\Phi}(k x)\right),
$$

is a norm that will be called s-norm on the Orlicz space $L_{\Phi}$.

Combing the definitions of various norms, the inequality

$$
\|x\|_{\Phi} \leq\|x\|_{\Phi, s} \leq\|x\|_{\Phi}^{o},
$$

holds. Denote

$$
L_{\Phi, s}=\left(L_{\Phi},\|\cdot\|_{\Phi, s}\right), E_{\Phi, s}=\left(E_{\Phi},\|\cdot\|_{\Phi, s}\right) .
$$

Definition 6 (see [4]). $s^{*}$ is an outer function that is conjugate to $s$ in the Hölder sense.

For any $x \in L_{\Phi, s}$ and $y \in L_{\Psi, s^{*}}$ the following Hölder inequality holds.

$$
\left|\int_{G} x(t) y(t) \mathrm{d} t\right| \leq\|x\|_{\Phi, s}\|y\|_{\Psi, s^{*}}
$$

Definition 7 (see [4]). For an outer function $s$ and its righthand derivative $s_{+}^{\prime}$, define

$$
\omega(v)=\int_{0}^{v} s_{+}^{\prime-1}(t) \mathrm{d} t,
$$

whenever $v \in[0,1]$.

For all $u \in[0, \infty), v \in[0, \infty)$, define

$$
\beta_{s}(u, v)=1-\omega\left(s_{+}^{\prime}(u)\right)-v s_{+}^{\prime}(u),
$$

and for all $x \in L_{\Phi, s} /\{(0)\}$,

$$
\begin{aligned}
k^{*}(x) & =\inf \left\{k>0: \beta_{s}\left(I_{\Phi}(k x), I_{\Psi}\left(p_{+}(k|x|)\right)\right) \leq 0\right\}, \\
k^{* *}(x) & =\sup \left\{k>0: \beta_{s}\left(I_{\Phi}(k x), I_{\Psi}\left(p_{+}(k|x|)\right)\right) \geq 0\right\} .
\end{aligned}
$$

Lemma 1 (see [4]). For any $x \in L_{\Phi, s} /\{0\}$, if and only if $k \in(0, \infty) \cap k(x)$, where $k(x)=\left[k^{*}(x), k^{* *}(x)\right]$ for short, we have

$$
\|x\|_{\Phi, s}=\frac{1}{k} s\left(I_{\Phi}(k x)\right) .
$$

Definition 8. We say that an Orlicz function $\Phi$ satisfies condition $\Delta_{2}\left(\Phi \in \Delta_{2}\right.$, for short $)$ if there exists $K>0$ and $u_{0}>0$, such that

$$
\Phi(2 u) \leq K \Phi(u), \quad|u| \geq u_{0} .
$$

Definition 9. Let $X$ be a Banach space. If $x_{n} \in S(X)$, $x \in S(X)$, and $x_{n} \longrightarrow^{w} x$ imply $x_{n} \longrightarrow x(n \longrightarrow \infty)$, then we say that $X$ has the Kadec-Klee property (see [5-7]).

Lemma 2. For any $f \in\left(L_{\Phi, s}\right)^{*}$ has a unique decomposition,

$$
f=\varphi+v\left(\varphi \in F, v \in\left(E_{\Phi, s}\right)^{*}\right),
$$

where $F$ is the set of all singular functionals on $E_{\Phi, s}$.

Lemma 3 (see [8]). If $\Phi$ is strictly convex, then for any $[a, b] \subset(0,1)$ and $D, \varepsilon>0$, there exists $\delta>0$, such that

$$
\Phi[\lambda u+(1-\lambda) v] \leq(1-\delta)[\lambda \Phi(u)+(1-\lambda) \Phi(v)],
$$

whenever $\lambda \in[a, b],|u| \leq D,|v| \leq D,|u-v| \geq \varepsilon$.

Lemma 4 (see [8]). A subset $A=\left\{x_{n}\right\} \subset L_{\Phi, s}$ is $L_{\Psi, s^{*}}$ weakly compact (i.e., there exists a subsequence $\left\{x_{n_{i}}\right\} \subset\left\{x_{n}\right\}$ and $x \in L_{\Phi, s}$, such that $v\left(x_{n_{i}}\right) \longrightarrow v(x)$ for each $\left.v \in L_{\Psi, s^{*}}\right)$ if

$$
\lim _{\lambda \rightarrow 0} \sup \frac{I_{\Phi}\left(\lambda x_{n}\right)}{\lambda}=0 .
$$

Lemma 5 (see [8]). For any $x_{n}, x \in L_{\Phi, s}$, assume that $I_{\Phi}\left(k_{n} x_{n}\right) \longrightarrow I_{\Phi}\left(k_{0} x\right)$ and $k_{n} x_{n}(t) \longrightarrow^{\mu} k_{0} x(t)$; if in addition, $\Phi \in \Delta_{2}$, then

$$
\left\|x_{n}-x\right\|_{\Phi, s} \longrightarrow 0
$$

\section{Main Results}

Theorem 1. Orlicz space $L_{\Phi, s}$ has the Kadec-Klee property if and only if

(1) $\Phi \in \Delta_{2}$

(2) $\Phi$ is strictly convex on $R$

Proof. Necessity. (i) If $\Phi \notin \Delta_{2}$, there exists $x \in S\left(L_{\Phi, s} / E_{\Phi, s}\right)$, such that $\lim _{n \longrightarrow \infty}\left\|x-x_{n}\right\|_{\Phi, s}=\theta(x)>0$, where $\theta(x)=\inf$ $\left\{\lambda>0, I_{\Phi}(x / \lambda)<\infty\right\}$ and $I_{\Phi}\left(x-x_{n}\right) \longrightarrow 0$ as $n \longrightarrow \infty$.

For any $n, m>0$, put

$G_{n}=\{t \in G:|x(t)| \leq n\}, G_{n, m}=\{t \in G: n \leq|x(t)| \leq m\}$. 
Using $\lim _{m \longrightarrow \infty}\left\|x \chi_{G_{n, m}}\right\|_{\Phi, s}$, we have

$$
\lim _{m \longrightarrow \infty}\left\|x \chi_{G_{n, m}}\right\|_{\Phi, s} \geq \theta(x) .
$$

Hence, for each $n \in N$, there exist $m_{n}>n>0$ satisfying $\left\|x \chi_{G_{n, m_{n}}}\right\|_{\Phi, s} \geq(\theta(x) / 2)$. Define $\overline{x_{n}}=x \chi_{G_{n, m_{n}}}$. Then,

$$
\left\|\overline{x_{n}}\right\|_{\Phi, s} \geq \frac{\theta(x)}{2} .
$$

Let us prove that $\overline{x_{n}} \longrightarrow^{w} 0$. For any $f=v+\varphi \in\left(L_{\Phi, s}\right)^{*}$, we have $\varphi\left(\overline{x_{n}}\right)=0$, thanks to $\overline{x_{n}} \in E_{\Phi, s}$ and

$$
f\left(\overline{x_{n}}\right)=v\left(\overline{x_{n}}\right)=\int_{G} v(t) \overline{x_{n}}(t) \mathrm{d} t=\int_{G_{n, m_{n}}} v(t) x(t) \mathrm{d} t .
$$
quality

Using $\int_{G} v(t) x(t) \mathrm{d} t<\infty$ and $\lim _{n \longrightarrow \infty} m\left(G_{n, m_{n}}\right)=0$, the

$$
\lim _{n \longrightarrow \infty} \int_{G_{n, m_{n}}} v(t) x(t)=0
$$

holds.

Put $y_{n}(t)=x(t)-\overline{x_{n}}(t)$. Then, $y_{n} \longrightarrow^{w} x$, and

$$
1 \geq\left\|y_{n}\right\|_{\Phi, s} \geq\left\|x \chi_{G \mid G_{n}}\right\|_{\Phi, s} \longrightarrow\|x\|_{\Phi, s}=1,
$$

that is $\left\|y_{n}\right\|_{\Phi, s}=1$. But,

$$
\left\|y_{n}-x\right\|_{\Phi, s}=\left\|\overline{x_{n}}\right\|_{\Phi, s} \geq \frac{\theta(x)}{2},
$$

this contract with that $L_{\Phi, s}$ has the Kadec-Klee property.

(ii) If $\Phi$ is not strictly convex, there exists $x \in S\left(L_{\Phi, s}\right)$, such that $x$ is not an extreme point, i.e., there exists $k_{0} \in\left[k^{*}(x), k^{* *}(x)\right], \quad$ such that $m\left(\left\{t \in G: \mid k_{0}\right.\right.$ $\left.\left.x(t) \mid \notin S_{\Phi}\right\}\right)>0$. Therefore, there exist $0<a<b$, such that

$$
\Phi(u)=A u+B,
$$

for some $A, B \in R^{+}$when $u \in(a, b)$.

Put

$$
\begin{aligned}
& G_{0}=\left\{t \in G:\left|k_{0} x(t)\right| \in(a, b)\right\}, \\
& G_{n}=\left\{t \in G_{0}:\left|k_{0} x(t)\right| \in\left(a+\frac{1}{n}, b-\frac{1}{n}\right)\right\} .
\end{aligned}
$$

Then, $\lim m\left(G_{n}\right)=m\left(G_{0}\right)$. Without loss of generality, we may assume that $x(t) \geqslant 0$ for each $t \in G_{0}$.

Therefore, there exists a $n_{0} \in N$, such that $m\left(G_{n_{0}}\right)>0$. Let $c=\left(1 / m\left(G_{n_{0}}\right)\right) \int_{G_{n_{0}}} x(t) \mathrm{d} t$. Then, $\left(a / k_{0}\right)<c<\left(b / k_{0}\right)$. Put $x_{0}(t)=c \chi_{G_{n}}+x(t) \chi_{G \mid G_{n_{0}}}$ and $\delta=\left(k_{0} / n_{0} b\right)$. Then, we have $k x(t) \in(a, b)$ for any $k \in\left(k_{0}-\delta, k_{0}+\delta\right)$ and $t \in G_{n_{0}}$. Hence,

$$
\begin{aligned}
I_{\Phi}(k x(t)) & =\int_{G_{n_{0}}} \Phi(k x(t)) \mathrm{d} t+\int_{G \mid G_{n_{0}}} \Phi(k x(t)) \mathrm{d} t \\
& =\int_{G_{n_{0}}}[A(k x(t))+B] \mathrm{d} t+\int_{G \mid G_{n_{0}}} \Phi(k x(t)) \mathrm{d} t \\
& =A k c m\left(G_{n_{0}}\right)+B m\left(G_{n_{0}}\right)+\int_{G \mid G_{n_{0}}} \Phi(k x(t)) \mathrm{d} t \\
& =\Phi(k c) m\left(G_{n_{0}}\right)+\int_{G \mid G_{n_{0}}} \Phi(k x(t)) \mathrm{d} t \\
& =I_{\Phi}\left(k x_{0}(t)\right),
\end{aligned}
$$

i.e., $\quad I_{\Phi}\left(k x_{0}(t)\right)=I_{\Phi}(k x(t))$. Then, we have that $\beta_{s}\left(I_{\Phi}\left(k x_{0}\right), I_{\Psi}\left(p_{+}\left(k\left|x_{0}\right|\right)\right)\right) \leq 0 \quad$ when $\quad \beta_{s}\left(I_{\Phi}(k x), I_{\Psi}\left(p_{+}\right.\right.$ $(k|x|))) \leq 0$ and $\beta_{s}\left(I_{\Phi}\left(k x_{0}\right), I_{\Psi}\left(p_{+}\left(k\left|x_{0}\right|\right)\right)\right) \geq 0$ when $\beta_{s}\left(I_{\Phi}(k x), I_{\Psi}\left(p_{+}(k|x|)\right)\right) \geq 0$. By formula (27), we have

$$
\|x\|_{\Phi, s}=\frac{1}{k_{0}} s\left(I_{\Phi}\left(k_{0} x(t)\right)\right)=\frac{1}{k_{0}} s\left(I_{\Phi}\left(k_{0} x_{0}(t)\right)\right)=\left\|x_{0}\right\|_{\Phi, s^{*}}
$$

Divide $G_{0}$ into two disjoint sets $G_{1}^{(1)}$ and $G_{1}^{(2)}$, such that

$$
m\left(G_{1}^{(1)}\right)=m\left(G_{1}^{(2)}\right)=\frac{1}{2} m\left(G_{n_{0}}\right),
$$

and divide $G_{1}^{(1)}$ and $G_{1}^{(2)}$ into two disjoint sets $G_{2}^{(1)}, G_{2}^{(2)}$ and $G_{2}^{(3)}, G_{2}^{(4)}$, respectively, such that

$$
m\left(G_{2}^{(1)}\right)=m\left(G_{2}^{(2)}\right)=m\left(G_{2}^{(3)}\right)=m\left(G_{2}^{(4)}\right)=\frac{1}{2^{2}} m\left(G_{n_{0}}\right) .
$$
that

By induction as above, we obtain a sequence $\left\{G_{n}^{(i)}\right\}$, such

$$
m\left(G_{n}^{(2 i-1)}\right)=m\left(G_{n}^{(2 i)}\right)=\frac{1}{2^{n}} m\left(G_{0}\right),
$$

for every $n \in N$ and $i=1,2, \ldots, 2^{n}$. Define

$$
x_{n}(t)=(c+\varepsilon) \chi_{\cup_{i=1}^{2 n-1} G_{n}^{(2 i-1)}}+(c-\varepsilon) \chi_{\cup_{i=1}^{2 n-1} G_{n}^{(2 i)}}+x(t) \chi_{G \mid G_{n_{0}}} .
$$
hold:

Since, for $k_{0} \in\left[k^{*}(x), k^{* *}(x)\right]$, the following equalities

$$
\begin{aligned}
I_{\Phi}\left(k_{0} x_{n}(t)\right)= & \int_{G_{1}^{(1)}} \Phi\left(k_{0}(c+\varepsilon)\right) \mathrm{d} t+\int_{G_{1}^{(2)}} \Phi\left(k_{0}(c-\varepsilon)\right) \mathrm{d} t \\
& +\int_{G \mid G_{n_{0}}} \Phi\left(k_{0} x(t)\right) \mathrm{d} t \\
= & \int_{G_{n_{0}}} \Phi\left(k_{0} c\right) \mathrm{d} t+\int_{G \mid G_{n_{0}}} \Phi\left(k_{0} x(t)\right) \mathrm{d} t=I_{\Phi}\left(k_{0} x_{0}(t)\right),
\end{aligned}
$$


whence $\|x\|_{\Phi, s}=\left\|x_{0}\right\|_{\Phi,} \quad s=\left(1 / k_{0}\right) s\left(I_{\Phi}\left(k_{0} x_{0}(t)\right)\right)=(1 /$ $\left.k_{0}\right) s\left(I_{\Phi}\left(k_{0} x_{n}(t)\right)\right)=\left\|x_{n}\right\|_{\Phi, s}$.

Let $\quad \overline{x_{n}}(t)=x_{n}(t)-x(t) \chi_{G \mid G_{n}}$; we get that $\lim _{\lambda \longrightarrow 0} \sup \left(I_{\Phi}\left(\lambda \overline{x_{n}}\right) / \lambda\right)=0$, without loss of generality, using Lemma 4 , we may assume that $\overline{x_{n}} \longrightarrow{ }^{L_{\Psi}}, s^{*} \bar{x}$ for some $\bar{x} \in L_{\Phi, s}$; using $\overline{x_{n}} \in E_{\Phi, s}$, we have that $\overline{x_{n}} \longrightarrow^{w} \bar{x}$ holds. Put $\tilde{x}=\bar{x}+x(t) \chi_{G \mid G}$. Then, $x_{n} \longrightarrow^{w} \tilde{x}$.

Let $y(t) \in S\left(L_{\Psi, s^{*}}\right)$ be a support functional of $x_{0}(t)$. Denote $\quad d=\left(1 / m\left(G_{n_{0}}\right)\right) \int_{G_{n_{0}}} y(t) \mathrm{d} t \quad$ and $\quad y_{0}(t)=$ $d \chi_{G_{n_{0}}}+y(t) \chi_{G \mid G_{n_{0}}}$. We get the following:

$$
\begin{aligned}
y_{0}\left(x_{0}\right) & =\int_{G} y_{0}(t) x_{0}(t) \mathrm{d} t \\
& =\int_{G_{n_{0}}} y_{0}(t) x_{0}(t) \mathrm{d} t+\int_{G \mid G_{n_{0}}} y(t) x(t) \mathrm{d} t \\
& =\int_{G_{n_{0}}} c d d t+\int_{G \mid G_{n_{0}}} y(t) x(t) \mathrm{d} t \\
& =c \int_{G_{n_{0}}} y(t) \mathrm{d} t+\int_{G \mid G_{n_{0}}} y(t) x(t) \mathrm{d} t \\
& =y\left(x_{0}\right)=\left\|x_{0}\right\|_{\Phi, s}=1,
\end{aligned}
$$

whence

$\left\|y_{0}\right\|_{\Psi, s^{*}}=\left\|y_{0}\right\|_{\Psi, s^{*}} \cdot\left\|x_{0}\right\|_{\Phi, s} \geq y_{0}\left(x_{0}\right)=y\left(x_{0}\right)=\|x\|_{\Phi, s}=1 . \quad\|y\|_{\Psi, s^{*}}=(1 / k) s^{*}\left(I_{\Psi}(k y)\right)$. Then,

$$
\begin{aligned}
\left\|y_{0}\right\|_{\Psi, s^{*}} & \leq \frac{1}{k} s^{*}\left(I_{\Psi}\left(k y_{0}(t)\right)\right)=\frac{1}{k} s^{*}\left(\int_{G} \Psi\left(k y_{0}(t)\right) \mathrm{d} t\right) \\
& =\frac{1}{k} s^{*}\left(\int_{G_{n_{0}}} \Psi(k d) \mathrm{d} t+\int_{G \mid G_{n_{0}}} \Psi(k y(t)) \mathrm{d} t\right) \\
& =\frac{1}{k} s^{*}\left(\int_{G_{n_{0}}}\left(\frac{1}{m\left(G_{n_{0}}\right)} \int_{G_{n_{0}}} \Psi(k y(t)) \mathrm{d} t\right) \mathrm{d} t+\int_{G \mid G_{n_{0}}} \Psi(k y(t)) \mathrm{d} t\right) \\
& =\frac{1}{k} s^{*}\left(I_{\Psi}(k y(t))\right)=1 .
\end{aligned}
$$

Hence, we have that $\left\|y_{0}\right\|_{\Psi, s^{*}}=1$ holds.

Since $\quad x_{n} \longrightarrow^{w} \tilde{x}, \quad\|\widetilde{x}\|_{\Phi, s} \leq \liminf _{n \rightarrow \infty}\left\|x_{n}\right\|_{\Phi, s}=1, \quad$ and $\|\widetilde{x}\|_{\Phi, s} \geq y_{0}(\widetilde{x})=1$, so $\|\widetilde{x}\|_{\Phi, s}=1$.

Furthermore,

$$
\left\|x_{n}-x_{m}\right\|_{\Phi, s}=2 \varepsilon\left\|\chi_{G_{1}^{(1)}}\right\|_{\Phi, s} .
$$

It follows that $x_{n}$ is not a Cauchy sequence, a contradiction.
Sufficiency. Assume that $s$ is an outer function, and Orlicz function $\Phi$ is strictly convex satisfying the $\Delta_{2}$ condition. Take $x_{n}, x \in S\left(L_{\Phi, s}\right)$ satisfying $\left\|x_{n}+x\right\|_{\Phi, s} \longrightarrow 2$ and $x_{n} \longrightarrow^{w} x$.

For any $x \in L_{\Phi, s}$, let $f(k)=(1 / k) s\left(I_{\Phi}(k x)\right)$ and $G_{a}=$ $\{t:|x(t)|>a\}$ for some $a>0$ with $m\left(G_{a}\right)>0$. By the definition of $s$, we have

$$
f(k)=\frac{1}{k} s\left(I_{\Phi}(k x)\right) \geq \frac{I_{\Phi}(k x)}{k} \geq \int_{G_{a}} \frac{\Phi(k x)}{k} \mathrm{~d} t \geq \int_{G_{a}} \frac{\Phi(k a)}{k} \mathrm{~d} t \geq \frac{\Phi(k a)}{k} m\left(G_{a}\right),
$$

and

$$
f(k)=\frac{1}{k} s\left(I_{\Phi}(k x)\right) \geq \frac{1}{k} .
$$

Since $\lim _{u \longrightarrow \infty}(\Phi(u) / u)=\infty$, we obtain $\lim _{k \rightarrow \infty} f$ $(k)=\infty$ and $\lim _{k \rightarrow 0} f(k)=\infty$, i.e., $k(x) \neq \varnothing$. Then, there exist $k_{n} \in\left[k^{*}\left(x_{n}\right), k^{* *}\left(x_{n}\right)\right]$, such that 


$$
\left\|x_{n}\right\|_{\Phi, s}=\frac{1}{k_{n}} s\left(I_{\Phi}\left(k_{n} x_{n}\right)\right)=1 .
$$

First, we will prove that $x_{n} \longrightarrow x$ in measure. Suppose that $k_{n} x_{n} \rightarrow k_{0} x$ as $n \longrightarrow \infty$, there exist $\varepsilon_{0}>0, \delta_{0}>0$, such that $m\left(\left\{t \in G:\left|k_{n} x_{n}-k_{0} x\right| \geq \varepsilon_{0}\right\}\right) \geq \delta_{0}$ for any $n \in N$.

(i) Assume that $k_{n}$ is bounded, and we have $\sup _{n>0} k_{n}=\bar{k}<\infty$.

Hence,

$$
0<\frac{k_{0}}{k_{0}+\bar{k}} \leq \frac{k_{0}}{k_{0}+k_{n}} ; \frac{k_{n}}{k_{0}+k_{n}} \leq \frac{\bar{k}}{k_{0}+\bar{k}}<1 .
$$

Using $k_{n}=s\left(I_{\Phi}\left(k_{n} x_{n}\right)\right)$, we have

$$
\begin{aligned}
\bar{k} & \geq k_{n}=s\left(I_{\Phi}\left(k_{n} x_{n}\right)\right) \\
& \geq \int_{G} \Phi\left(k_{n} x_{n}(t)\right) \mathrm{d} t \\
& \geq \int_{\left\{t \in G:\left|k_{n} x_{n}(t)\right|>k\right\}} \Phi\left(k_{n} x_{n}(t)\right) \mathrm{d} t \\
& \geq \Phi(k) m\left(\left\{t \in G:\left|k_{n} x_{n}(t)\right|>k\right\}\right) .
\end{aligned}
$$

Then, there exists $k \in N$, such that $m\left(\left\{t \in G:\left|k_{n} x_{n}(t)\right|>k\right\}\right) \leq(\bar{k} / \Phi(k))<\left(\delta_{0} / 3\right)$. Set

$$
G_{n}=\left\{\begin{array}{cc} 
& \left|k_{n} x_{n}(t)\right| \leq k \\
t \in G: & \left|k_{0} x(t)\right| \leq k \\
& \left|k_{n} x_{n}(t)-k_{0} x(t)\right| \geq \varepsilon_{0}
\end{array}\right\} .
$$

Then, $m\left(G_{n}\right)<\left(\delta_{0} / 3\right)$. In virtue of Lemma 3 , there exists $\delta_{1}>0$, such that

$$
\begin{aligned}
\Phi\left(\frac{k_{0} k_{n}}{k_{0}+k_{n}}\left(x_{n}(t)+x(t)\right)\right) \leq & \left(1-\delta_{1}\right)\left[\frac{k_{0}}{k_{0}+k_{n}} \Phi\left(k_{n} x_{n}(t)\right)\right. \\
& \left.+\frac{k_{n}}{k_{0}+k_{n}} \Phi\left(k_{0} x(t)\right)\right] .
\end{aligned}
$$

Whenever $t \in G_{n}$. Combining with the definition of the functions $s$ and $\Phi$ as well as with formulas (42) and (45), we obtain

$$
\begin{aligned}
2 \leftarrow\left\|x_{n}+x\right\|_{\Phi, s} \leq & \frac{k_{0}+k_{n}}{k_{0} k_{n}} s\left(I_{\Phi}\left(\frac{k_{0} k_{n}}{k_{0}+k_{n}}\left(x_{n}(t)+x(t)\right)\right)\right) \\
\leq & \frac{k_{0}+k_{n}}{k_{0} k_{n}} s\left(\int_{G_{n}} \Phi\left(\frac{k_{0} k_{n}}{k_{0}+k_{n}}\left(x_{n}(t)+x(t)\right)\right) \mathrm{d} t+\int_{G \mid G_{n}} \Phi\left(\frac{k_{0}+k_{n}}{k_{0} k_{n}}\left(x_{n}(t)+x(t)\right)\right) \mathrm{d} t\right) \\
\leq & \frac{k_{0}+k_{n}}{k_{0} k_{n}} s\left(\left(1-\delta_{1}\right)\left(\frac{k_{0}}{k_{0}+k_{n}} \int_{G_{n}} \Phi\left(k_{n} x_{n}(t)\right) \mathrm{d} t+\frac{k_{n}}{k_{0}+k_{n}} \int_{G_{n}} \Phi\left(k_{0} x(t)\right) \mathrm{d} t\right)\right. \\
& +\frac{k_{0}}{k_{0}+k_{n}} \int_{G \mid G_{n}} \Phi\left(k_{n} x_{n}(t)\right) \mathrm{d} t+\frac{k_{n}}{k_{0}+k_{n}} \int_{G \mid G_{n}} \Phi\left(k_{0} x(t)\right) \mathrm{d} t \\
\leq & \frac{k_{0}+k_{n}}{k_{0} k_{n}}\left(s \left(\frac{k_{0}}{k_{0}+k_{n}} \int_{G} \Phi\left(k_{n} x_{n}(t)\right) d t+\frac{k_{n}}{k_{0}+k_{0}} \int_{G} \Phi\left(k_{0} x(t)\right) d t\right.\right. \\
& \left.-s\left(\delta_{1}\left(\frac{k_{0}}{k_{0}+k_{n}} \int_{G_{n}} \Phi\left(k_{n} x_{n}(t)\right) d t+\frac{k_{n}}{k_{0}+k_{n}} \int_{G_{n}} \Phi\left(k_{0} x(t)\right) d t\right)\right)\right] \\
\leq & 2-\frac{k_{0}+k_{n}}{k_{0} k_{n}} s\left(\delta_{1}\left(\frac{k_{0}}{k_{0}+k_{n}} \int_{G_{n}} \Phi\left(k_{n} x_{n}(t)\right) d t+\frac{k_{n}}{k_{0}+k_{n}} \int_{G_{n}} \Phi\left(k_{0} x(t)\right) d t\right)\right) \\
\leq & 2-\delta_{1}\left(\frac{1}{k_{n}} \int_{G_{n}} \Phi\left(k_{n} x_{n}(t)\right) \mathrm{d} t+\frac{1}{k_{0}} \int_{G_{n}} \Phi\left(k_{0} x(t)\right) \mathrm{d} t\right) \\
\leq & 2-\frac{2 \delta_{1}}{\bar{k}} \frac{\int_{G_{n}}\left(\Phi\left(k_{n} x_{n}(t)\right)+\Phi\left(k_{0} x(t)\right)\right) \mathrm{d} t}{2} \leq 2-\frac{2 \delta_{1}}{\bar{k}} \int_{G_{n}} \Phi\left(\frac{k_{n} x_{n}(t)-k_{0} x(t)}{2}\right) \mathrm{d} t<2-\frac{2 \delta_{1}}{\bar{k}} \Phi\left(\frac{\varepsilon_{0}}{2}\right) \frac{\delta_{0}}{3},
\end{aligned}
$$


A contradiction. Consequently, $k_{n} x_{n} \longrightarrow^{\mu} k_{0} x$ as $n \longrightarrow \infty$.

(ii) If the sequence $\left(k_{n}\right)$ is not bounded and without loss of generality, we may assume that $\lim _{n \longrightarrow+\infty}$ $k_{n}=+\infty$ and set $y_{n}=\left(x_{n}+x / 2\right), l_{n}=\left(k_{n} k_{0} / k_{n}+\right.$ $\left.k_{0}\right)$; then, $y_{n} \longrightarrow^{w} x, \lim _{n \longrightarrow \infty}\left\|y_{n}\right\|_{\Phi, s}=1$, and the following inequalities hold.

$$
\begin{aligned}
2 \lim _{n \longrightarrow \infty}\left\|y_{n}\right\|_{\Phi, s} \leqslant & \lim _{n \rightarrow \infty} \frac{1}{l_{n}} s\left(I_{\Phi}\left(l_{n} y_{n}\right)\right) \\
& =\lim _{n \longrightarrow \infty} \frac{k_{n}+k_{0}}{k_{n} k_{0}} s\left(I_{\Phi}\left(\frac{k_{0}}{k_{n}+k_{0}} k_{n} x_{n}+\frac{k_{n}}{k_{n}+k_{0}} k_{0} x\right)\right) \\
& \leqslant \lim _{n \longrightarrow \infty} \frac{1}{k_{n}}\left(1+I_{\Phi}\left(k_{n} x_{n}\right)\right)+\frac{1}{k_{0}}\left(1+I_{\Phi}\left(k_{0} x\right)\right) \\
& =2,
\end{aligned}
$$

It is clear that the sequence $\left(l_{n}\right)$ is bounded, and

$$
\lim _{n \longrightarrow \infty} l_{n}=\lim _{n \longrightarrow \infty} \frac{k_{n} k_{0}}{k_{n}+k_{0}}=\lim _{n \longrightarrow \infty} \frac{k_{0}}{1+\left(k_{0} / k_{n}\right)}=k_{0} .
$$

Similar as the proof above, we have $l_{n} x_{n} \longrightarrow^{\mu} k_{0} x$. Second, we will show that $\lim k_{n}=k_{0}$. We only need to prove that $k_{n} x_{n}(t)$ converges $k_{0} x(t)$ weakly star.

For any $\varepsilon>0$ and $v \in E_{\Psi, s^{*}}$, using that $v$ has absolutely continuous norm, there exists $\delta>0, E \subset G$, and $m(E)<\delta$, such that

$$
\left\|v \chi_{E}\right\|_{\Psi, s^{*}}<\frac{\varepsilon}{3 \overline{\bar{k}}}
$$

By Yegolov's theorem, there exists $e_{0} \subset G$ with $m\left(e_{0}\right)<\delta$ for which $k_{n} x_{n}(t) \longrightarrow k_{0} x(t)$ uniformly $t \in G \mid e_{0}$.

Hence, there is $n_{0} \in N$ satisfying

$$
\left\|\left(k_{n} x_{n}-k_{0} x\right) \chi_{G \mid e_{0}}\right\|_{\Phi, s} \leqslant \frac{\varepsilon}{3\|v\|_{\Psi, s^{*}}},
$$

Whenever $n \geq n_{0}$ and $t \in G \mid e_{0}$.

So,

$$
\begin{aligned}
& \left|\int_{G} k_{n} x_{n}(t) v(t) \mathrm{d} t-\int_{G} k_{0} x(t) v(t) \mathrm{d} t\right| \\
& \leq \int_{G \mid e_{0}}\left|k_{n} x_{n}(t)-k_{0} x(t) \| v(t)\right| \mathrm{d} t+\int_{e_{0}}\left|k_{n} x_{n}(t) v(t)\right| \mathrm{d} t+\int_{e_{0}}\left|k_{0} x(t) v(t)\right| \mathrm{d} t \\
& \leq\left\|\left(k_{n} x_{n}-k_{0} x\right) \chi_{G \mid e_{0}}\right\|_{\Phi, s}\|v\|_{\Psi, s^{*}}+\bar{k}\left\|x_{n}\right\|_{\Phi, s}\left\|v \chi_{e_{0}}\right\|_{\Psi, s^{*}}+\bar{k}\|x\|_{\Phi, s}\left\|v \chi_{e_{0}}\right\|_{\Psi, s^{*}} \\
& \leq \frac{\varepsilon}{3}+\frac{\varepsilon}{3}+\frac{\varepsilon}{3} \\
& =\varepsilon
\end{aligned}
$$

i.e., $k_{n} x_{n} \longrightarrow^{w^{*}} k_{0} x$. By $x_{n} \longrightarrow^{w} x$, we can get that $k_{n} \longrightarrow k_{0}$ holds.

Finally, by formula (41), we have $s^{-1}\left(k_{n}\right)=I_{\Phi}\left(k_{n} x_{n}\right)$ and $s^{-1}\left(k_{0}\right)=I_{\Phi}\left(k_{0} x\right)$. Hence, we get that $I_{\Phi}\left(k_{n} x_{n}\right)$ $\longrightarrow I_{\Phi}\left(k_{0} x\right)$ holds. In virtue of Lemma 5 , we obtain $\left\|x_{n}-x\right\|_{\Phi, s} \longrightarrow 0$.

\section{Data Availability}

No data were used to support this study.

\section{Conflicts of Interest}

The authors declare that there are no conflicts of interest.

\section{Acknowledgments}

This work was supported by the National Natural Science Foundation of China (11871181) and the Natural Science Foundation of Heilongjiang Province (A2018006).

\section{References}

[1] M. A. Krasnoselskii and Y. B. Rutickii, Convex Function and Orlicz Spaces, Nordhoff, Groningen, Netherlands, 1961.

[2] Y. Cui, L. Duan, H. Hudzik, and M. Wisła, "Basic theory of p-Amemiya norm in Orlicz spaces $(1 \leq p \leq \infty)$ : extreme points and rotundity in orlicz spaces equipped with these norms," Nonlinear Analysis, vol. 69, no. 5, pp. 1796-1816, 2008.

[3] H. Hudzik and L. Maligranda, "Amemiya norm equals Orlicz norm in general," Indagationes Mathematicae, vol. 11, no. 4, pp. 573-585, 2000.

[4] M. Wisła, "Orlicz spaces equipped with s-norms," Journal of Mathematical Analysis and Applications, vol. 483, no. 2, pp. 123659-123689, 2019.

[5] Y. Cui, P. Foralewski, H. Hudzik, and R. Kaczmarek, "Kadecklee properties of Orlicz-Lorentz sequence spaces equipped with the Orlicz norm," Positivity, vol. 25, pp. 1-22, 2021.

[6] Jean Saint Raymond, "Kadec-klee property and fixed points," Journal of Functional Analysis, vol. 266, no. 8, pp. 5429-5438, 2014. 
[7] T. Dominguez, H. Hudzik, G. López, M. Mastylo, and B. Sims, "Complete characterization of Kadec-Klee properties in Orlicz spaces," Houston Journal of Mathematics, vol. 29, no. 4, pp. 1027-1044, 2003.

[8] S. Chen, "Geometry of Orlicz spaces," Dissertationes Mathematicae, vol. 356, Warszawa, 1996. 